\title{
Downregulation of DEC1 by RNA interference attenuates ischemia/reperfusion-induced myocardial inflammation by inhibiting the TLR4/NF-кB signaling pathway
}

\author{
WEIPAN XU, KAI ZHANG, YI ZHANG, SHANXUE MA and DAOQUN JIN \\ Department of Cardiology, Huangshi Central Hospital, Affiliated Hospital of Hubei Polytechnic University, \\ Edong Healthcare Group, Huangshi, Hubei 435000, P.R. China
}

Received October 21, 2019; Accepted March 5, 2020

DOI: $10.3892 /$ etm.2020.8706

\begin{abstract}
Inflammation has been implicated in the pathogenesis of myocardial ischemia/reperfusion (I/R) injury (MIRI). Previous studies have confirmed that deleted in esophageal cancer 1 (DEC1) is an important transcription factor in inflammation. However, the role of DEC1 in MIRI remains unclear. The present study aimed to determine whether the downregulation of DEC1 by RNA interference alleviated inflammation to protect against MIRI. Adult Sprague-Dawley rats $(n=48)$ were randomly divided into four groups: Sham; I/R; adenovirus expressing green fluorescent protein control (Ad-G-Control); and DEC1-targeting RNA interference (Ad-G-DEC1) groups. Following gene delivery 4 days later, the rat myocardial I/R model was established and myocardial enzymes [creatine kinase (CK) and lactate dehydrogenase (LDH)] were detected. Hematoxylin and eosin (H\&E) staining was performed to evaluate the myocardial damage and the infarct area was assessed using Evans Blue/triphenyltetrazolium chloride staining. The inflammatory mediators interleukin (IL)- $\beta$ and tumor necrosis factor (TNF)- $\alpha$ were also detected using ELISA kits to assess the inflammatory response. Finally, western blotting and reverse transcription-quantitative PCR were used to analyze the expression levels of associated proteins and mRNAs. Ad-G-DEC1 RNA interference markedly decreased DEC1 expression levels. In addition, following the downregulation of DEC1 expression, the infarct size, $\mathrm{CK}, \mathrm{LDH}$, Toll-like receptor (TLR) 4, NF- $\kappa B$, IL- $\beta$ and TNF- $\alpha$ levels were all significantly decreased. In conclusion, the results of the present study suggested that the downregulation of DEC1 may decrease
\end{abstract}

Correspondence to: Dr Daoqun Jin, Department of Cardiology, Huangshi Central Hospital, Affiliated Hospital of Hubei Polytechnic University, Edong Healthcare Group, 141 Tianjin Road, Huangshi, Hubei 435000, P.R. China

E-mail: jindaoqunhs@163.com

Key words: deleted in esophageal cancer 1, RNA interference, myocardial ischemia/reperfusion injury, inflammation, Toll-like receptor $4 / \mathrm{NF}-\kappa \mathrm{B}$ the inflammation by suppressing the TLR4/NF- $\mathrm{BB}$ signaling pathway, which may represent a therapeutic target for MIRI.

\section{Introduction}

Ischemic heart disease is a leading cause of morbidity and mortality worldwide (1). The most effective clinical intervention for myocardial ischemia is timely revascularization (2). However, myocardial ischemia/reperfusion (I/R) caused by revascularization can also promote further myocardial damage (3). The pathophysiological mechanism of myocardial I/R injury (MIRI) is complicated; however, numerous studies have revealed that the inflammatory response serves an important role in I/R-induced myocardial damage (4-6). Thus, determining novel approaches to decrease the inflammation may lead to effective treatments for MIRI.

Deleted in esophageal cancer 1 (DEC1) was first identified in human embryonic cartilage tissue and it has since been confirmed to function as a basic helix-loop-helix transcription factor that can regulate transcription in various types of cell, including hepatocyte and nerve cells (7). The expression levels of DEC1 have been identified to significantly increase in response to environmental stimuli, and DEC1 has been demonstrated to be involved in various physiological activities, such as chondrocyte differentiation (8), lipid metabolism (9), cell proliferation (10) and the immune response (11). Increasing evidence has suggested that DEC1 is also an important transcription factor in inflammation (12-14). The continuous activation of DEC1 and the consequent elevation of the inflammatory cascade events has served as a crucial pathogenic mechanism in a wide range of human inflammatory diseases, such as periodontitis (12), autoimmune encephalomyelitis (13) and rheumatoid arthritis (15).

It is well established that the activation of the Toll-like receptor 4 (TLR4)/NF- $\kappa B$ signaling pathway induces the transcription of numerous cytokines to promote inflammatory responses $(16,17)$. For example, Zhang et al (12) reported that DEC1 modulated Porphyromonas gingivalis-induced periodontitis via the TLR4/NF- $\mathrm{BB}$ signaling pathway. In fact, the excessive activation of the TLR4/NF- $\mathrm{KB}$ inflammatory pathway has been identified to be closely associated with the development of MIRI $(18,19)$. However, whether the activation 
of TLR4/NF- $\kappa \mathrm{B}$ could also be triggered by DEC1 during MIRI remains largely unknown. Thus, further study into the physiological function and underlying mechanisms of DEC1 in MIRI is required. The present study demonstrated that the genetic silencing of DEC1 using RNA interference technology reduced inflammation in MIRI by repressing the TLR4/NF-kB signaling pathway. Thus, the present study provided a novel insight into the potential mechanisms of DEC1 in MIRI and may provide a novel therapeutic target for preventing MIRI.

\section{Materials and methods}

Animal studies. The procedures for the experiments and animal care were approved by the Animal Care and Use Committee of Hubei Polytechnic University and conformed to the Guide for the Care and Use of Laboratory Animals produced by the National Institutes of Health (20). A total of 48 adult male Sprague-Dawley rats (age, 8 weeks; weight, about $250 \mathrm{~g}$ ) were purchased from Vital River Laboratory Animal Technology Co., Ltd. and housed at $23 \pm 2^{\circ} \mathrm{C}$ with $50 \%$ relative humidity, with a 12-h light/dark cycle and ad libitum access to food and water.

Small interfering RNA (siRNA) interference. The adenovirus (RNA interference) containing siRNA against DEC1 was synthesized and generated with a pBHGlox_E1,3Cre plasmid (Microbix Biosystems) using the AdMax system (Microbix Biosystems) according to the manufacturer's protocols. A scrambled sequence (500 $\mu$ 1; Sangon Biotech Co., Ltd.) was used as the negative control. 293T cells (cat. no. CRL-1573; American Type Culture Collection; $4 \times 10^{5}$ cells $/ \mathrm{ml}$ ) were used to package and amplify the adenoviruses $(500 \mu \mathrm{l})$ with Lipofectamine $^{\mathrm{TM}} 2000$ (Invitrogen; Thermo Fisher Scientific, Inc.) in DMEM (Gibco; Thermo Fisher Scientific, Inc.), supplemented with $10 \%$ FBS (Gibco; Thermo Fisher Scientific, Inc.) at $37^{\circ} \mathrm{C}$ in a $5 \% \mathrm{CO}_{2}$ incubator for $10-15$ days. After the majority of 293T cells exhibited typical cytopathic effects, samples were frozen-thawed at $-70^{\circ} \mathrm{C} / 37^{\circ} \mathrm{C}$ three times and centrifuged at $7,000 \mathrm{xg}$ at $4^{\circ} \mathrm{C}$ for $5 \mathrm{~min}$. Virus supernatants were subsequently collected. The final virus concentration was $1 \times 10^{11}$ plaque-forming units, as using the endpoint dilution method (21).

Establishment of MIRI model rats. The 48 healthy male $\mathrm{SD}$ rats were divided into four groups ( $\mathrm{n}=12 /$ group): The sham group (Sham); I/R group (I/R); adenovirus expressing green fluorescent protein Control group (Ad-G-Control); and DEC1-targeting RNA interference group (Ad-G-DEC1). The rat MIRI model was prepared in the I/R, Ad-G-Control and Ad-G-DEC1 groups. Briefly, sodium pentobarbital $(30 \mathrm{mg} / \mathrm{kg}$; intraperitoneal) was used to anesthetize the rats and open the chest. A volume of $80 \mu \mathrm{l}$ Ad-G-Control or Ad-G-DEC1 diluted in saline $\left(1 \times 10^{10} \mathrm{PFU} / \mathrm{ml} ; 10\right.$ times diluted) was injected into the heart wall of 8-week-old rats. A total of 4 days later, the rats were re-anesthetized, the rats' chests were reopened and the left anterior descending artery (LAD) was ligated using a 6-0 silk suture. Following $30 \mathrm{~min}$ of ligation, the circulation was restored for $\sim 4$ h by removing the silk suture; when the rat's limbs demonstrated slight movement ( $\sim 70 \mathrm{~min}$ intervals), the rats were re-anesthetized to keep them under anesthetic during this procedure. Post-reperfusion, the rats were sacrificed by jugular vein injection of potassium chloride $(75 \mathrm{mg} / \mathrm{kg})$. Death was confirmed using a standard body part II-lead electrocardiogram. Both blood $(5 \mathrm{ml})$ and part of the anterior wall of the left ventricular myocardium near the cardiac apex were subsequently harvested from the rats. The sham groups served as the control and no occlusion of the LAD was performed.

Determination of myocardial enzymes. Harvested blood was centrifuged at $500 \mathrm{xg}$ for $5 \mathrm{~min}$ at $4^{\circ} \mathrm{C}$ to obtain serum, which was subsequently used to measure myocardial enzyme levels, including creatine kinase (CK; cat. no. QS1107) and lactate dehydrogenase (LDH; cat. no. QS1001) using commercially available biochemical kits (Beijing Solarbio Science \& Technology Co., Ltd.), according to the manufacturers' protocols. The data obtained are presented as U/l.

Detection of myocardial infarct area (IA)/area at risk (AAR). Evans Blue/triphenyltetrazolium chloride (TTC) staining was used to determine the IA following MIRI. In brief, the LADs of the rats were immediately ligated following $4 \mathrm{~h}$ of reperfusion and $\sim 1 \mathrm{ml}$ Evans blue (Sigma-Aldrich; Merck KGaA) was intravenously injected to distinguish non-ischemic and risk areas. Next, rats were sacrificed by injecting $75 \mathrm{mg} / \mathrm{kg}$ potassium chloride into the jugular vein, after which hearts were removed, frozen at $-20^{\circ} \mathrm{C}$ for $10 \mathrm{~min}$ and sliced into $2 \mathrm{~mm}$ thick sections. Sections were subsequently stained with $1.5 \%$ TTC (Sigma-Aldrich; Merck KGaA) for 15 min at room temperature. The risk area was stained red, whilst the IA appeared white. Image-Pro Plus 5.0 software (Media Cybernetics, Inc.) was used to calculate the AAR (red + white) and IA (white). The IA/AAR ratio was calculated for quantitative analysis.

Histological examination. The harvested hearts were fixed in $10 \%$ formalin for $48 \mathrm{~h}$ at room temperature and embedded in paraffin. Paraffin-embedded tissues were sliced into $4-\mu \mathrm{m}$-thick sections and stained with hematoxylin and eosin (hematoxylin staining, $5 \mathrm{~min}$ at room temperature; eosin staining, 2 min at room temperature). Light microscopy (magnification, $\mathrm{x} 200$ ) was used to observe the morphological changes in the myocardial tissue following reperfusion in each group.

Reverse transcription-quantitative PCR (RT- $q P C R)$. RT-qPCR was used to detect mRNA levels as previously described (19). In detail, total RNA was extracted from the harvested hearts using TRIzol ${ }^{\circledR}$ reagent (Invitrogen; Thermo Fisher Scientific, Inc.) and reverse-transcribed into cDNA using the SuperScript IV Reverse Transcriptase kit (Thermo Fisher Scientific, Inc.) at $37^{\circ} \mathrm{C}$ for $60 \mathrm{~min}$ according to the manufacturer's protocol. qPCR was subsequently performed using the SYBR Green Master Mix kit (Thermo Fisher Scientific, Inc.) on the 7500 ABI Prism system in accordance with the manufacturer's protocol. The following thermocycling conditions were used for the qPCR: Initial denaturation at $95^{\circ} \mathrm{C}$ for $10 \mathrm{~min}$; followed by 40 of cycles of denaturation at $95^{\circ} \mathrm{C}$ for $30 \mathrm{sec}$, annealing at $60^{\circ} \mathrm{C}$ for $30 \mathrm{sec}$ and elongation at $72^{\circ} \mathrm{C}$ for $30 \mathrm{sec}$. Data were analyzed using the $2^{-\Delta \Delta C q}$ method (22). The following primer pairs were used for the qPCR: TLR4 forward, 5'-AGTGTA TCGGTGGTCAGTGTGCT-3' and reverse, 5'-AAACTCCAG 


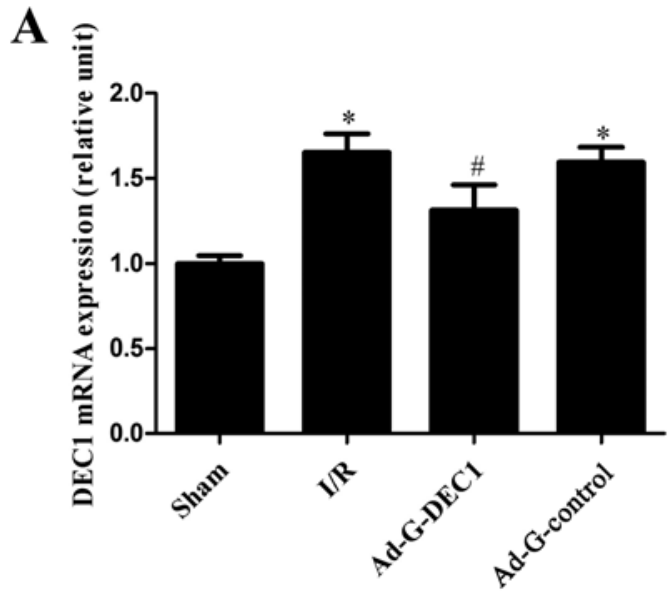

B
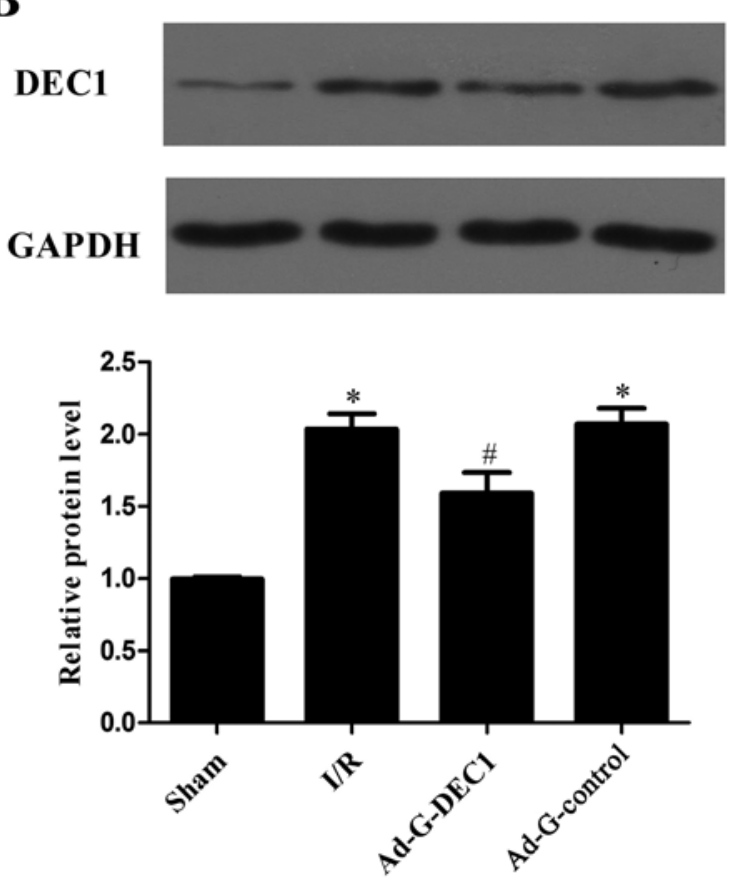

Figure 1. Delivery of DEC1-targeting RNA interference decreases the increased expression levels of DEC1 induced by I/R. (A) Reverse transcription-quantitative PCR and (B) western blotting were used to analyze the mRNA or protein expression levels, respectively, of DEC1. Data are presented as the mean \pm standard deviation $(\mathrm{n}=5)$. ${ }^{*} \mathrm{P}<0.05$ vs. sham group; ${ }^{\#} \mathrm{P}<0.05$ vs. the $\mathrm{I} / \mathrm{R}$ group. DEC1, deleted in esophageal cancer $1 ; \mathrm{I} / \mathrm{R}$, ischemia-reperfusion; Ad, adenovirus.

CCACACATTCC-3'; NF- $\kappa$ B forward, 5'-AAGCACAGATAC CACTAAGACGCA -3' and reverse, 5'-TTCAGCCTCATA GAAGCCATCC-3'; DEC1 forward, 5'-GTCTGTGAGTCA CTCTTCAG-3' and reverse, 5'-GAGTCTAGTTCTGTTTGA AGG-3'; and GAPDH forward, 5'-TGGCCTTCCGTGTTC CTAC-3' and reverse, 5'-GAGTTGCTGTTGAAGTCGCA-3'. The mRNA expression levels of DEC1, TLR4 and NF- $\kappa B$ were normalized to GAPDH.

Western blotting. Western blotting was performed to detect the protein expression levels of DEC1, TLR4, NF- $\mathrm{B}$ and GAPDH in the harvested hearts. Briefly, total protein was extracted using the protein extraction kit (cat. no. P0028; Beyotime Institute of Biotechnology). Total protein $(40 \mu \mathrm{g})$ was quantified using a bicinchoninic acid assay (Beyotime
Institute of Biotechnology) and separated via 10\% SDS-PAGE. The separated proteins were subsequently transferred onto PVDF membranes electrophoretically (EMD Millipore). The membranes were blocked with 5\% non-fat dry milk in PBS with $0.05 \%$ Tween-20 for $2 \mathrm{~h}$ at room temperature. Samples were then incubated with the following primary antibodies at $4^{\circ} \mathrm{C}$ overnight: Anti-DEC1 (1:600; cat. no. sc-101023; Santa Cruz Biotechnology, Inc.), anti-TLR4 (1:500; cat. no. sc-10741; Santa Cruz Biotechnology, Inc.), anti-NF-кB (1:600; cat. no. sc-514451; Santa Cruz Biotechnology, Inc.) and anti-GADPH (1:1,000; cat. no. sc-48166; Santa Cruz Biotechnology, Inc.). Following the primary antibody incubation, the membranes were washed 3 times with TBS with $0.05 \%$ Tween and incubated with horseradish peroxidase (HRP)-conjugated anti-mouse (1:5,000; cat. no. AS014), HRP-conjugated anti-goat (1:5,000; cat. no. AS064) or HRP-conjugated anti-rabbit (1:5,000; cat. no. AS063; all, ABclonal Biotech Co., Ltd.) IgG secondary antibodies for $2 \mathrm{~h}$ at room temperature. Finally, protein bands were visualized using an enhanced chemiluminescence system (Thermo Fisher Scientific, Inc.). Quantity One 1-D software (version 4.6.9; Bio-Rad Laboratories, Inc.) was used for densitometric analysis.

ELISAs. ELISA kits (R\&D Systems, Inc.) were used to determine the concentrations of interleukin (IL)-1 $\beta$ (cat. no. SRLB00) and tumor necrosis factor (TNF)- $\alpha$ (cat. no. SRTA00) in the harvested hearts, according to the manufacturer's protocol.

Statistical analysis. Statistical analysis was performed using SPSS 21.0 software (IBM Corp.) and data are presented as the mean \pm standard deviation $(n=5)$. Statistical differences between two groups were determined using a Student's t-test, whereas statistical differences between more than two groups were analyzed using a one-way ANOVA, followed by a Tukey's post hoc test for multiple comparisons. $\mathrm{P}<0.05$ was considered to indicate a statistically significant difference.

\section{Results}

Delivery of DEC1-targeting RNA interference decreases the increased DECl expression levels induced by $I / R$. Following reperfusion, compared with the sham group, the mRNA and protein expression levels of DEC1 were significantly increased in the $\mathrm{I} / \mathrm{R}$ group $(\mathrm{P}<0.05$; Fig. $1 \mathrm{~A}$ and $\mathrm{B})$. Notably, following the delivery of the DEC1-targeting RNA inference, the expression levels of DEC1 were significantly decreased compared with the I/R and Ad-G-Control groups ( $\mathrm{P}<0.05$; Fig. 1).

Delivery of DEC1-targeting RNA interference decreases the concentration of serum marker enzymes. LDH and CK are commonly used as molecular markers for myocardial injury (23). The activities of CK and LDH were significantly increased in the I/R group compared with the sham group $(\mathrm{P}<0.05$; Fig. $2 \mathrm{~A}$ and $\mathrm{B})$; however, following the delivery of DEC1-targeting RNA interference, the concentration of CK and LDH in the Ad-G-DEC1 group was significantly decreased compared with the $\mathrm{I} / \mathrm{R}$ and Ad-G-Control groups ( $\mathrm{P}<0.05$; Fig. 2).

Decreased expression levels of DEC1 decreases the IA. To quantitatively determine the extent of myocardial injury, 

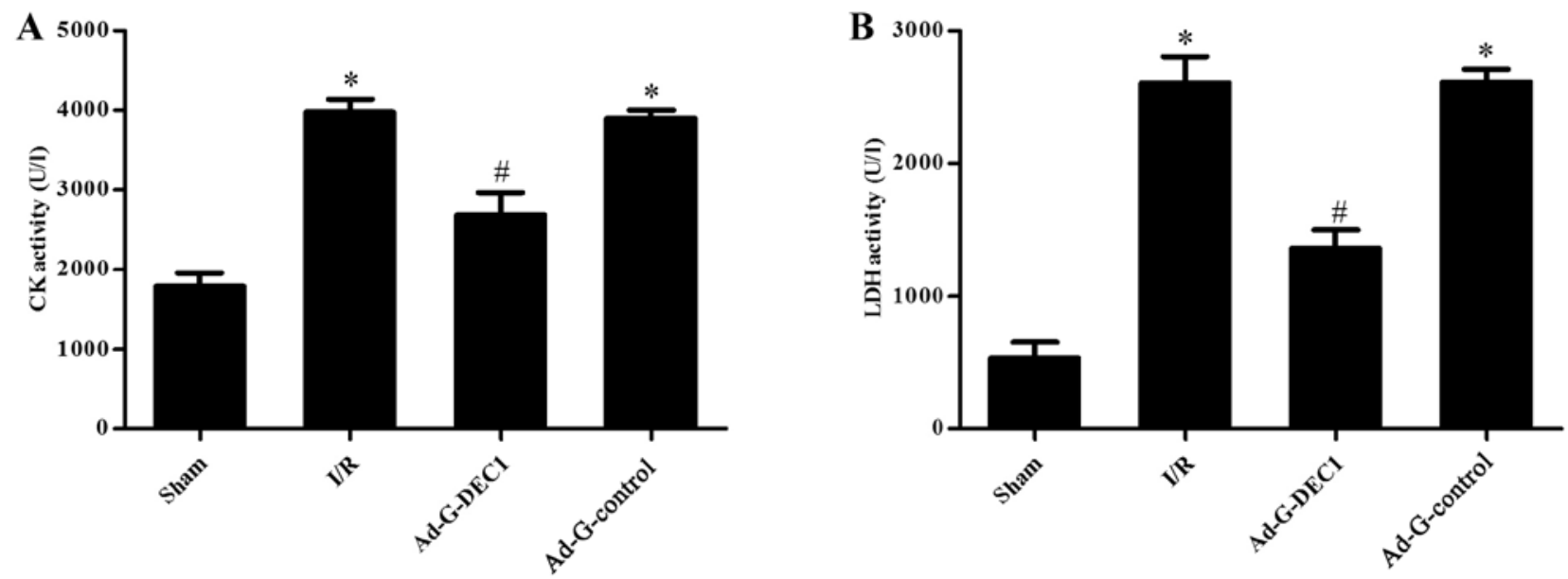

Figure 2. Delivery of DEC1-targeting RNA interference decreases the levels of serum marker enzymes. (A) CK and (B) LDH activities were detected using commercially available biochemical kits and demonstrated significantly decreased activites following the gene transfer of Ad-G-DEC1. Data are presented as the mean \pm standard deviation $(\mathrm{n}=5)$. ${ }^{*} \mathrm{P}<0.05$ vs. sham group. ${ }^{\sharp} \mathrm{P}<0.05$ vs. the $\mathrm{I} / \mathrm{R}$ group. DEC1, deleted in esophageal cancer 1 ; $\mathrm{CK}$, creatine kinase; $\mathrm{LDH}$, lactate dehydrogenase; I/R, ischemia-reperfusion; Ad, adenovirus.

A

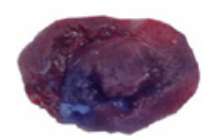

Sham

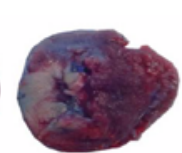

$\mathbf{I} / \mathbf{R}$

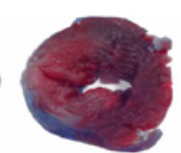

Ad-G-DEC1 Ad-G-control
B

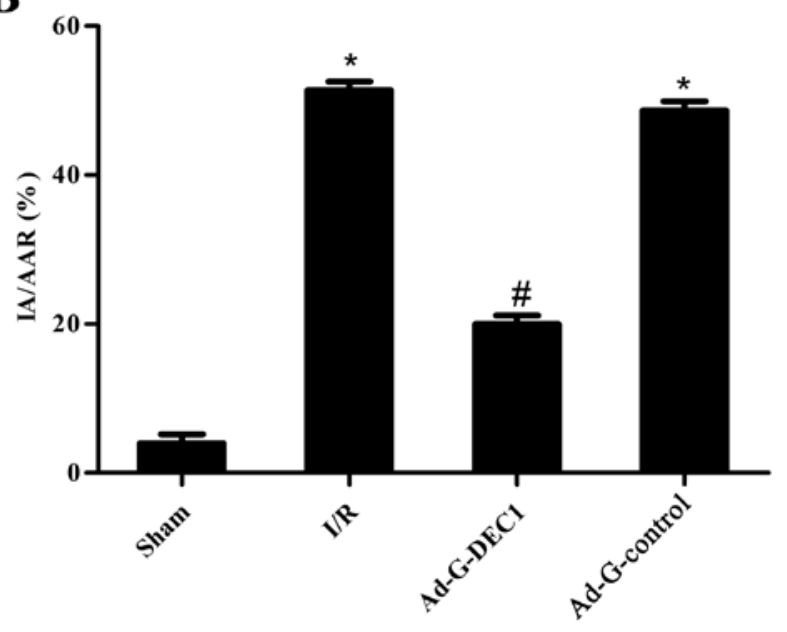

Figure 3. Downregulation of DEC1 expression decreases infarct size. (A) Representative pictures of Evans and triphenyltetrazolium chloride staining in each group. (B) IA/AAR was used to evaluate the IA. Data are presented as the mean \pm standard deviation $(n=5)$. ${ }^{*} \mathrm{P}<0.05$ vs. sham group. ${ }^{\text {"}} \mathrm{P}<0.05$ vs. the I/R group; IA, infarct area; AAR, area at risk; DEC1, deleted in esophageal cancer $1 ; \mathrm{I} / \mathrm{R}$, ischemia-reperfusion; Ad, adenovirus.

the IA/AAR was calculated. The IA/AAR was identified to be $56.4 \pm 6.2 \%$ in the I/R group (Fig. 3). Notably, the downregulation of DEC1 expression in Ad-G-DEC1 group exerted a cardioprotective effect by reducing IA/AAR during MIRI compared with the I/R group $(\mathrm{P}<0.05$; Fig. 3$)$. However, no significant effect on IA/AAR was observed in the Ad-G-Control group compared with the I/R group (P>0.05; Fig. 3).
Histopathological evaluation of the heart tissue. In the I/R group, the myocardial fibers were observed to be disorganized and ruptured, exhibiting signs of edema (Fig. 4). Notably, the transfection of the DEC1-targeting RNA interference vector partially decreased the myocardial damage. Furthermore, no significant effect was observed in the histological morphology of the heart tissue in the Ad-G-Control group compared with the I/R group (Fig. 4).

Determination of the levels of $I L-1 \beta$ and TNF- $\alpha$. ELISAs were used to detect the concentrations of IL- $1 \beta$ and TNF- $\alpha$ in the harvested specimens. It was revealed that the levels of IL-1 $\beta$ and TNF- $\alpha$ were significantly increased in the I/R group compared with the sham group, whereas the downregulation of DEC1 expression significantly decreased their levels compared with the I/R and Ad-G-Control groups ( $\mathrm{P}<0.05$; Fig. 5).

Downregulation of DECl expression inhibits the TLR4/NF- $\mathrm{B} B$ signaling pathway. To investigate the underlying mechanisms of DEC1-induced I/R injury, the expression levels of TLR4 and NF- $\mathrm{KB}$ were determined. I/R significantly increased the mRNA and protein expression levels of TLR4 and NF- $\mathrm{KB}$ compared with the sham group $(\mathrm{P}<0.05$; Fig. 6A-C). However, the delivery of the DEC1-targeting RNA interference at the onset of I/R significantly decreased the expression levels of TLR4 and NF-kB compared with those in the I/R and Ad-G-Control groups. Therefore, these results suggested that I/R injury may be suppressed via the downregulation of DEC1, which may occur through its ability to inhibit the TLR4/NF- $\mathrm{B}$ signaling pathway.

\section{Discussion}

MIRI is an important cause of myocardial damage and it is an unsolved problem in clinical practice (24). Inflammation has been identified to serve a crucial role in MIRI and a persistent proinflammatory reaction promotes a series of complications following reperfusion therapy $(25,26)$. Therefore, effectively 

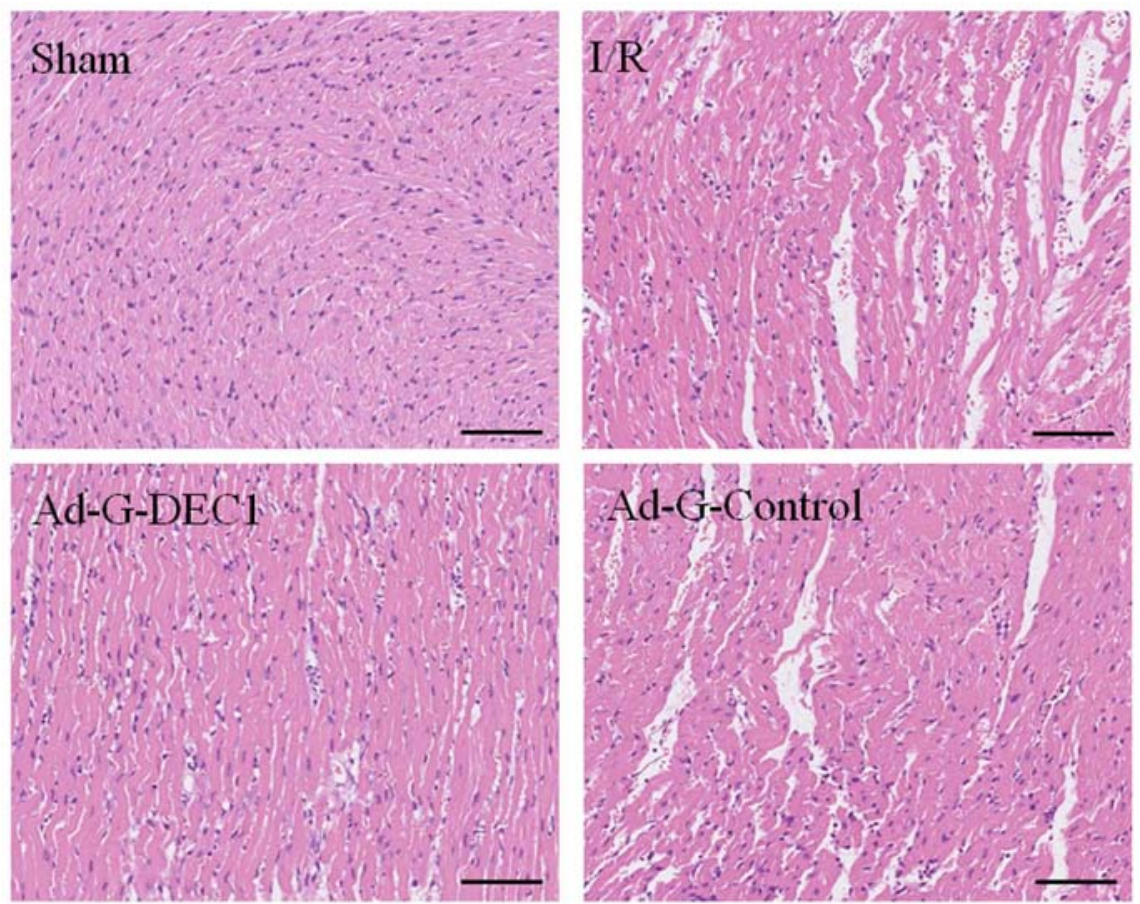

Figure 4. Histological investigations of the heart tissues. The myocardial fibers in the sham group were arranged in an orderly fashion. In the Ad-G-Control I/R groups, the myocardial fibers were disorganized and ruptured alongside the presence of edema. Transfection of the DEC1-targeting RNA interference vector partially decreased the myocardial damage. Scale bar=100 $\mu \mathrm{m}$; magnification, x200. DEC1, deleted in esophageal cancer 1; I/R, ischemia-reperfusion; Ad, adenovirus.

A

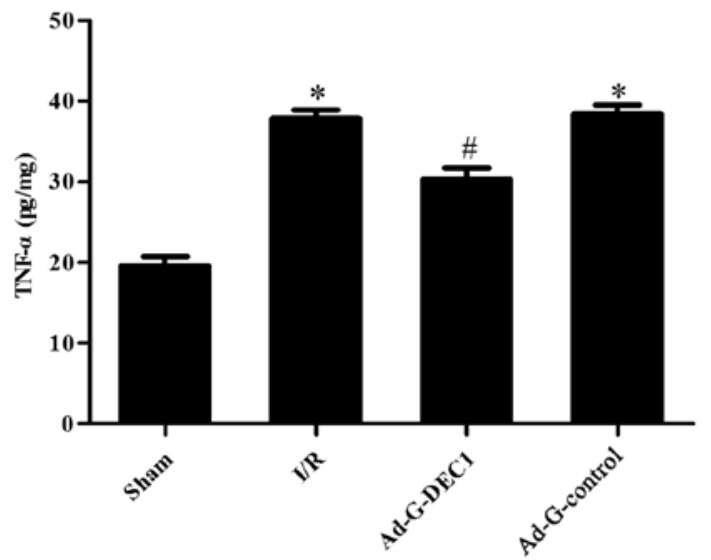

B

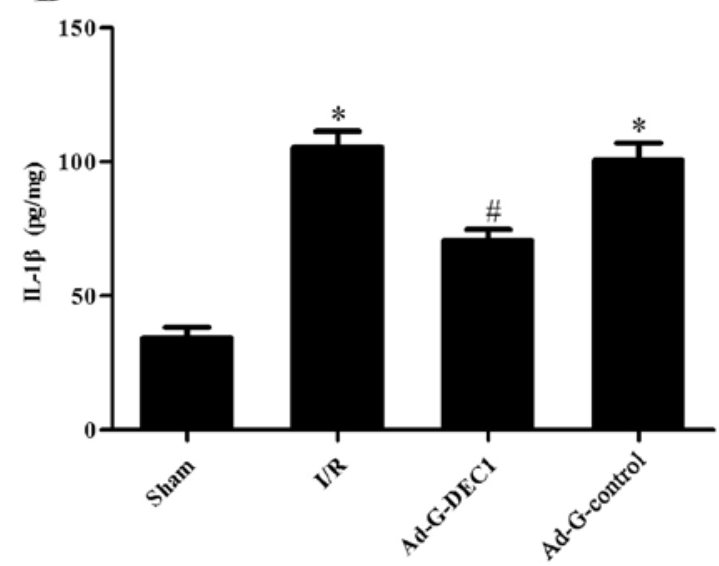

Figure 5. Downregulation of DEC1 expression decreases inflammatory responses. ELISAs were used to analyze the concentrations of (A) TNF- $\alpha$ and (B) IL-1 $\beta$ in the harvested hearts. Inflammatory factors were significantly increased in I/R group; however, the downregulation of DEC 1 decreased TNF- $\alpha$ and IL-1 $\beta$ levels. Data are presented as the mean \pm standard deviation $(n=5)$. ${ }^{*} \mathrm{P}<0.05$ vs. sham group; ${ }^{\#} \mathrm{P}<0.05$ vs. the $\mathrm{I} / \mathrm{R}$ group. $\mathrm{DEC} 1$, deleted in esophageal cancer 1 ; $\mathrm{I} / \mathrm{R}$, ischemia-reperfusion; IL-1 $\beta$, interleukin-1 $\beta$; TNF- $\alpha$, tumor necrosis factor- $\alpha$; Ad, adenovirus.

decreasing inflammation may be an important therapeutic target to improve the outcomes for MIRI. A previous study has found that DEC1 served an important role in various physiological functions, including cell differentiation, circadian rhythm regulation, hypoxia and inflammatory responses (27). Thus, in the past decade, DEC1-mediated inflammatory activation has become an important topic of research $(12,14)$; however, it is unclear whether there is an association between DEC1-mediated inflammatory activation and MIRI. In the present study, it was observed that the downregulation of DEC1 by RNA interference decreased MIRI, which is char- acterized by the decreased release of LDH/CK and infarct area. In addition, DEC1 silencing inhibited the protein expression levels of inflammation-related cytokines, such as IL-1 $\beta$ and TNF- $\alpha$. In the mechanistic studies, it was observed that the downregulation of DEC1s significantly inhibited the TLR4/NF- $\kappa \mathrm{B}$ signaling pathway. The aforementioned results therefore indicated that DEC1 silencing may possess a marked ability to ameliorate I/R-induced damage and inflammation by inhibiting the TLR4/NF- $\mathrm{BB}$ signaling pathway.

TLR4 is a major cell surface initiator of inflammatory responses (28). Accumulating evidence has suggested that 


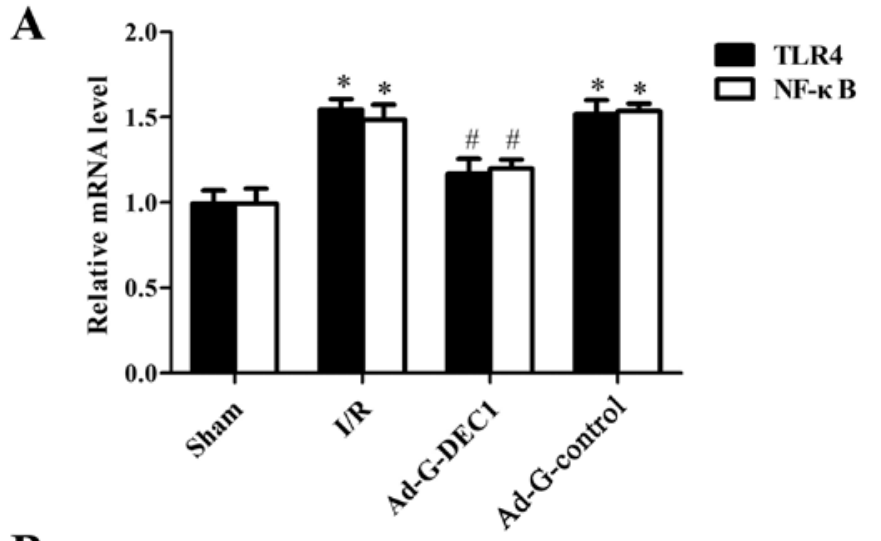

B

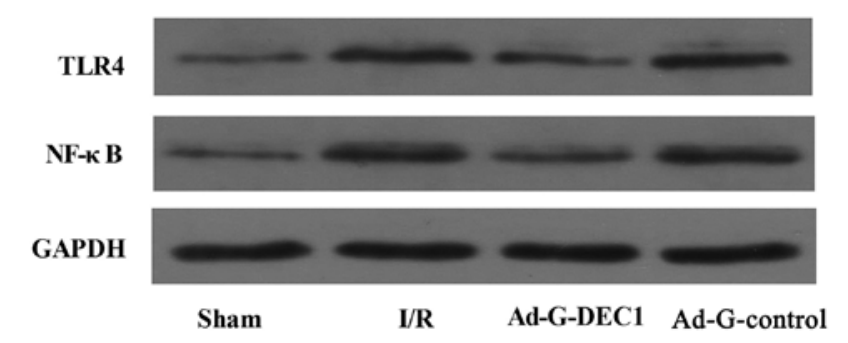

C

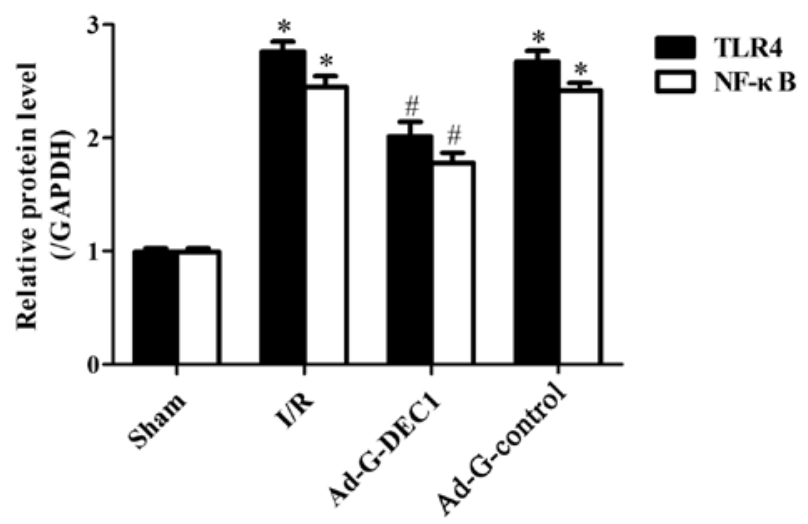

Figure 6. Downregulation of DEC1 expression inhibits the TLR4/NF- $\kappa B$ signaling pathway. mRNA and protein expression levels of TLR4 and NK- $\kappa \mathrm{B}$ were analyzed using (A) reverse transcription-quantitative PCR and (B and C) western blotting respectively. The downregulation of DEC1 expression inhibited the expression levels of TLR4 and NF- $\mathrm{B}$ mRNA and protein. Data are presented as the mean \pm standard deviation $(n=5)$. ${ }^{*} \mathrm{P}<0.05$ vs. sham group; ${ }^{\text {P }}<0.05$ vs. the I/R group. DEC1, deleted in esophageal cancer 1 ; I/R, ischemia-reperfusion; TLR4, Toll-like receptor 4; Ad, adenovirus.

TLR4-mediated signal transduction is activated during the process of MIRI $(29,30)$. Notably, Zhang et al (12) identified that the inflammation-associated transcription factor DEC1 regulated the cellular responses in $P$. gingivalis infection by promoting the production of IL- $1 \beta$ and TNF- $\alpha$ through the activation of TLR4. Meanwhile, the downregulation of DEC1 expression also decreased their expression levels and decrease the severity of periodontitis (12). With regards to the possible involvement of DEC1 in TLR4-associated inflammation, it was thus hypothesized that DEC1 may also serve a proinflammatory role in response to an I/R insult. Consistent with the aforementioned data, the results of the present study indicated that the DEC1-associated inflammatory signaling pathway was activated in the process of MIRI and inhibiting DEC1 expres- sion via RNA interference decreased the expression levels of IL-1 $\beta$, TNF- $\alpha$ and TLR4, which weakened the myocardial damage observed. Consequently, the present study provided evidence to suggest that DEC1-mediated TLR4 activation may participate in myocardial I/R; however, the potential mechanisms remain unknown.

$N F-\kappa B$ has a broad role in inflammation and other natural and pathological processes; its important role in the cardiovascular system following TLR4 activation been well established $(31,32)$. Notably, numerous studies have provided evidence that the TLR4/NF- $\mathrm{B}$ signaling pathway served an important role in the pathological process of MIRI $(18,19)$. For example, Olkkonen et al (15) indicated that the DEC gene enhanced NF- $\kappa \mathrm{B}$ binding to DNA to increase IL-1 $\beta$ expression levels in human fibroblasts. Therefore, it was necessary to investigate whether DEC1-mediated TLR4 signal transduction in MIRI was activated synergistically with $\mathrm{NF}-\kappa \mathrm{B}$; the data from the present study demonstrated that DEC1 silencing also significantly decreased the expression levels of $\mathrm{NF}-\kappa \mathrm{B}$.

The cytokine cascade in the inflammatory processes is a highly complex system (33). IL-1 $\beta$ and TNF- $\alpha$ are both proinflammatory cytokines, which have been previously implicated in the pathophysiology of MIRI (34). In addition, DEC1 has been confirmed to be an important transcription factor in inflammation by activating immune cells $\left(\mathrm{CD} 4^{+}\right.$ $\mathrm{T}$ cells) and controlling the expression of multiple immunomodulatory genes, such as IL-1 $\beta$ and TNF- $\alpha(12,14)$. Martínez-Llordella et al (13) also reported that DEC1-deficient mice exhibited an increased production of IL-10, but no effect on IFN- $\gamma$ levels. Based on these results, DEC1 was silenced and the concentrations of IL- $1 \beta$ and TNF- $\alpha$ were detected in the myocardium following $\mathrm{I} / \mathrm{R}$ in the present study. The results revealed that $\mathrm{DEC} 1$ silencing exerted protective effects against I/R injury by inhibiting the production of IL-1 $\beta$ and TNF- $\alpha$. IL- $\beta$ and NF- $\kappa B$ have been identified to independently contribute to the increased expression of DEC1, an effect that is dependent upon the PI3K/AKT signaling pathways, amongst others $(14,15)$. Taken together, these results suggested that the downregulation of DEC1 may not only decrease the levels of IL-1 $\beta$ and TNF- $\alpha$ directly, but it may also disturb the positive feedback loop between these inflammatory factors and DEC1.

In conclusion, the results of the present study demonstrated that the downregulation of DEC1 expression alleviated MIRI by attenuating inflammation in a TLR4/NF- $\kappa \mathrm{B}$-dependent manner. However, multiple other signaling pathways are considered to be involved in the pathophysiological processes of MIRI. Thus, further studies are required to investigate whether there are any other signaling pathways involved in the regulation over DEC1 in MIRI. Taken together, the present results suggested that $\mathrm{DEC} 1$ may be a prospective therapeutic option for MIRI.

\section{Acknowledgements}

Not applicable.

\section{Funding}

The present study was supported by the Hubei Provincial Natural Science Foundation of China (grant no. 2017CFB211) 
and the Health Commission of Hubei Province Scientific Research Project (grant no. WJ2019Q011).

\section{Availability of data and materials}

The datasets used and/or analyzed during the current study are available from the corresponding author on reasonable request.

\section{Authors' contributions}

WPX and KZ wrote the manuscript, interpreted the data and performed the experiments. YZ and SXM acquired and analyzed the data. WPX and DQJ performed the literature search, designed the study and revised the manuscript. All authors read and approval the final manuscript.

\section{Ethics approval and consent to participate}

The procedures for the experiments and animal care were approved by the Animal Care and Use Committee of Hubei Polytechnic University and conformed to the Guide for the Care and Use of Laboratory Animals produced by the National Institutes of Health.

\section{Patient consent for publication}

Not applicable.

\section{Competing interests}

The authors declare that they have no competing interests.

\section{References}

1. Nelson CP, Goel A, Butterworth AS, Kanoni S, Webb TR Marouli E, Zeng L, Ntalla I, Lai FY, Hopewell JC, et al Association analyses based on false discovery rate implicate new loci for coronary artery disease. Nat Genet 49: 1385-1391, 2017.

2. Godoy LC, Lawler PR, Farkouh ME, Hersen B, Nicolau JC and Rao V: Urgent revascularization strategies in patients with diabetes mellitus and acute coronary syndrome. Can J Cardiol 35 993-1001, 2019.

3. Shen Y, Liu X, Shi J and Wu X: Involvement of Nrf2 in myocardial ischemia and reperfusion injury. Int J Biol Macromol 125 496-502, 2019.

4. Wu MY, Yiang GT, Liao WT, Tsai AP, Cheng YL, Cheng PW, Li CY and Li CJ: Current mechanistic concepts in ischemia and reperfusion injury. Cell Physiol Biochem 46: 1650-1667, 2018.

5. Ruan Z, Wang S, Yu W and Deng F: LncRNA MALAT1 aggravates inflammation response through regulating PTGS2 by targeting miR-26b in myocardial ischemia-reperfusion injury. Int J Cardiol 288: 122, 2019.

6. Yao L, Chen H, Wu Q and Xie K: Hydrogen-rich saline alleviates inflammation and apoptosis in myocardial I/R injury via PINK-mediated autophagy. Int J Mol Med 44: 1048-1062, 2019.

7. Sato F, Kohsaka A, Bhawal UK and Muragaki Y: Potential roles of Dec and Bmall genes in interconnecting circadian clock and energy metabolism. Int J Mol Sci 19: pii: E781, 2018.

8. Liu Q, Wu Y, Seino H, Haga T, Yoshizawa T, Morohashi S and Kijima $\mathrm{H}$ : Correlation between DEC1/DEC2 and epithelial-mesenchymal transition in human prostate cancer PC-3 cells. Mol Med Rep 18: 3859-3865, 2018.

9. Noshiro M, Kawamoto T, Nakashima A, Ozaki N, Ueno T, Saeki M, Honda K, Fujimoto K and Kato Y: Deficiency of the basic helix-loop-helix transcription factor DEC1 prevents obesity induced by a high-fat diet in mice. Genes Cells, Jul 3, 2018 (Epub ahead of print).
10. Jia $Y, H u R$, Li P, Zheng Y, Wang $Y$ and Ma X: DEC1 is required for anti-apoptotic activity of gastric cancer cells under hypoxia by promoting Survivin expression. Gastric Cancer 21: 632-642, 2018.

11. Camponeschi A, Todi L, Cristofoletti C, Lazzeri C, Carbonari M, Mitrevski M, Marrapodi R, Del Padre M, Fiorilli M, Casato M and Visentini M: DEC1/STRA13 is a key negative regulator of activation-induced proliferation of human B cells highly expressed in anergic cells. Immunol Lett 198: 7-11, 2018.

12. Zhang F, Suzuki M, Kim IS, Kobayashi R, Hamada N, Sato F and Bhawal UK: Transcription factor DEC1 is required for maximal experimentally induced periodontal inflammation. J Periodontal Res 53: 883-893, 2018.

13. Martínez-Llordella M, Esensten JH, Lipsky RH, Marini A, Chen J, Mughal M, Mattson MP, Taub DD and Bluestone JA: CD28-inducible transcription factor DEC1 is required for efficient autoreactive CD4+ T cell response. J Exp Med 210: 1603-1619, 2013.

14. Bhawal UK, Ito Y, Tanimoto K, Sato F, Fujimoto K, Kawamoto T, Sasahira T, Hamada N, Kuniyasu H, Arakawa H, et al: IL-1 $\beta$-mediated up-regulation of DEC1 in human gingiva cells via the Akt pathway. J Cell Biochem 113: 3246-3253, 2012.

15. Olkkonen J, Kouri VP, Hynninen J, Konttinen YT and Mandelin J: Differentially expressed in chondrocytes 2 (DEC2) increases the expression of IL-1 $\beta$ and Is abundantly present in synovial membrane in rheumatoid arthritis. PLoS One 10: e0145279, 2015.

16. Zusso M, Lunardi V, Franceschini D, Pagetta A, Lo R, Stifani S, Frigo AC, Giusti P and Moro S: Ciprofloxacin and levofloxacin attenuate microglia inflammatory response via TLR4/NF-kB pathway. J Neuroinflammation 16: 148, 2019.

17. Rahimifard M, Maqbool F, Moeini-Nodeh S, Niaz K, Abdollahi M, Braidy N, Nabavi SM and Nabavi SF: Targeting the TLR4 signaling pathway by polyphenols: A novel therapeutic strategy for neuroinflammation. Ageing Res Rev 36: 11-19, 2017.

18. Yuan L, Dai X, Fu H, Sui D, Lin L, Yang L, Zha P, Wang X and Gong G: Vaspin protects rats against myocardial ischemia/reperfusion injury (MIRI) through the TLR4/NF- $\mathrm{B}$ signaling pathway. Eur J Pharmacol 835: 132-139, 2018.

19. Guo X, Jiang H, Yang J, Chen J, Yang J, Ding JW, Li S, Wu H and Ding HS: Radioprotective $105 \mathrm{kDa}$ protein attenuates ischemia/reperfusion-induced myocardial apoptosis and autophagy by inhibiting the activation of the TLR4/NF- $\mathrm{B}$ signaling pathway in rats. Int J Mol Med 38: 885-893, 2016.

20. Bayne K: Revised guide for the care and use of laboratory animals available. American physiological society. Physiologist 39: 199, 208-211, 1996

21. Mittereder N, March KL and Trapnell BC: Evaluation of the concentration and bioactivity of adenovirus vectors for gene therapy. J Virol 70: 7498-7509, 1996.

22. Livak JK and Schmittgen TD: Analysis of relative gene expression data using real-time quantitative PCR and the 2(-Delta Delta C(T)) method. Methods 25: 402-408, 2001.

23. Yang J, Fan ZX, Yang J, Ding J, Yang C and Chen L: microRNA-22 attenuates myocardial ischemia-reperfusion injury via an anti-inflammatory mechanism in rats. Exp Ther Med 12: 3249-3255, 2016

24. Neri M, Riezzo I, Pascale N, Pomara C and Turillazzi E: Ischemia/reperfusion injury following acute myocardial infarction: A critical issue for clinicians and forensic pathologists. Mediators Inflamm 2017: 7018393, 2017.

25. Chorawala MR, Prakash P, Doddapattar P, Jain M, Dhanesha N and Chauhan AK: Deletion of extra domain A of fibronectin reduces acute myocardial ischaemia/reperfusion injury in hyperlipidaemic mice by limiting thrombo-inflammation. Thromb Haemost 118: 1450-1460, 2018.

26. Hernandez-Resendiz S, Chinda K, Ong SB, Cabrera-Fuentes H, Zazueta $\mathrm{C}$ and Hausenloy DJ: The role of redox dysregulation in the inflammatory response to acute myocardial ischaemia-reperfusion injury-adding fuel to the fire. Curr Med Chem 25: 1275-1293, 2018.

27. Ivanov SV, Salnikow K, Ivanova AV, Bai L and Lerman MI: Hypoxic repression of STAT1 and its downstream genes by a pVHL/HIF-1 target DEC1/STRA13. Oncogene 26: 802-812, 2007.

28. Knowlton AA: Paying for the tolls: The high cost of the innate immune system for the cardiac myocyte. Adv Exp Med Biol 1003: 17-34, 2017

29. Fang $\mathrm{Y}$ and $\mathrm{Hu} \mathrm{J}$ : Toll-like receptor and its roles in myocardial ischemic/reperfusion injury. Med Sci Monit 17: RA100-RA109, 2011. 
30. Xue J, Ge H, Lin Z, Wang H, Lin W, Liu Y, Wu G, Xia J and Zhao Q: The role of dendritic cells regulated by HMGB1/TLR4 signalling pathway in myocardial ischaemia reperfusion injury. J Cell Mol Med 23: 2849-2862, 2019.

31. Su Q, Li L, Sun Y, Yang H, Ye Z and Zhao J: Effects of the

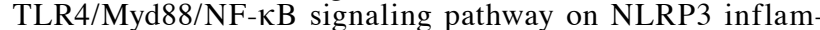
masome in coronary microembolization-induced myocardial injury. Cell Physiol Biochem 47: 1497-1508, 2018.

32. Tang ZH, Peng J, Ren Z, Yang J, Li TT, Li TH, Wang Z, Wei DH, Liu LS, Zheng XL and Jiang ZS: New role of PCSK9 in atherosclerotic inflammation promotion involving the TLR4/NF- $\mathrm{KB}$ pathway. Atherosclerosis 262: 113-122, 2017.
33. Chousterman BG, Swirski FK and Weber GF: Cytokine storm and sepsis disease pathogenesis. Semin Immunopathol 39: 517-528, 2017.

34. Ma C, Xu Z and Lv H: Low n-6/n-3 PUFA ratio improves inflammation and myocardial ischemic reperfusion injury. Biochem Cell Biol 97: 621-629, 2019.

(i) $(9$ This work is licensed under a Creative Commons cc) Attribution-NonCommercial-NoDerivatives 4.0 International (CC BY-NC-ND 4.0) License. 